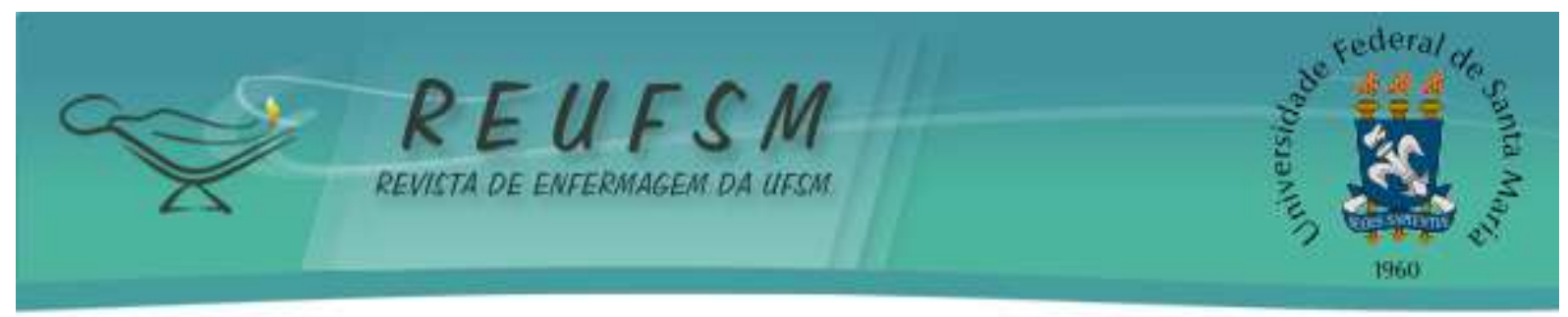

ARTIGO ORIGINAL

\title{
PERCEPÇÃO DE USUÁRIOS HOSPITALIZADOS SOBRE O ATENDIMENTO NOS SERVIÇOS DE SAÚDE
}

\section{PERCEPTION OF HOSPITALIZED USERS ON THE CARE IN HEALTH SERVICES \\ PERCEPCIÓN DE LOS USUARIOS HOSPITALIZADOS SOBRE LOS ATENDIMIENTOS EN SERVICIOS DE SALUD}

\author{
Pâmela Guimarães Siqueira ${ }^{1}$ \\ Rosângela Marion da Silva² \\ Carmem Lúcia Colomé Beck ${ }^{3}$ \\ Francine Cassol Prestes ${ }^{4}$ \\ Denise de Oliveira Vedootto ${ }^{5}$ \\ Thiana Sebben Pasa ${ }^{6}$
}

\section{Doi: $10.5902 / 2179769222355$}

RESUMO: Objetivo: conhecer a percepção de usuários hospitalizados sobre o atendimento nos serviços de saúde. Método: pesquisa qualitativa, descritiva e exploratória, realizada em uma unidade de Clinica Cirúrgica, no período de outubro a novembro de 2014. Os dados foram obtidos por meio de entrevistas semiestruturadas com usuários internados e analisados a partir da análise temática. Resultados: os resultados foram discutidos a partir das categorias: o caminhar nos serviços de saúde; e realidades da Atenção Primária à Saúde e da hospitalar. Identificou-se que os usuários percebem que o atendimento recebido nos serviços de saúde é dificultado pela falta de estrutura dos mesmos, pela comunicação deficiente ou inexistente e pela falta de humanização na assistência. Conclusão: Frente às dificuldades percebidas nos serviços de atenção primária à saúde, os usuários buscam atendimento em instituições hospitalares, situação que desfavorece o estabelecimento e a articulação das redes de atenção à saúde.

Descritores: Enfermagem; Humanização da assistência; Assistência integral à saúde; Serviços de saúde.

ABSTRACT: Aim: To know the perception of hospitalized patients about the care in health services. Method: qualitative, descriptive and exploratory research, carried out in a Surgical Clinic unit, from October to November 2014. Data were collected through semistructured interviews with hospitalized users and analyzed from the thematic analysis. Results: The results were discussed from the following categories: walking in the health services; and realities of the primary health care and of the hospital. It was identified that users perceive that the care received in health services is hampered by the lack of

\footnotetext{
${ }^{1}$ Enfermeira. Especializanda do Programa de Residência Multiprofissional pela Universidade Federal de Santa Maria (UFSM). Santa Maria/Rio Grande do Sul(RS)/Brasil. E-mail: pamelaguimaraessiqueira@gmail.com

2 Enfermeira. Doutora em Ciências. Docente da graduação do curso de Enfermagem e da Pós-graduação do Programa de Pós-Graduação em Enfermagem da UFSM. Santa Maria/RS/Brasil. E-mail: cucasma@terra.com.br

${ }^{3}$ Enfermeira. Doutora em Enfermagem. Docente da graduação do curso de Enfermagem e da Pós-graduação do Programa de Pós-Graduação em Enfermagem da UFSM. Santa Maria/RS/Brasil. E-mail: carmembeck@gmail.com 4 Enfermeira. Doutoranda em Enfermagem pelo PPGEnf/UFSM. Santa Maria/RS/Brasil. E-mail: francinecassol@gmail.com

5 Enfermeira. Mestranda em Enfermagem pelo PPGEnf/UFSM. Santa Maria/RS/Brasil. E-mail: dvedootto@bol.com.br

6 Enfermeira na Unidade de Cirurgia Geral- Serviço de Internação do HUSM. Especialista em Gestão em Enfermagem UAB-UNIFESP. Mestre em Enfermagem pelo PPGEnf /UFSM. E-mail:thianasp@hotmail.com
} 


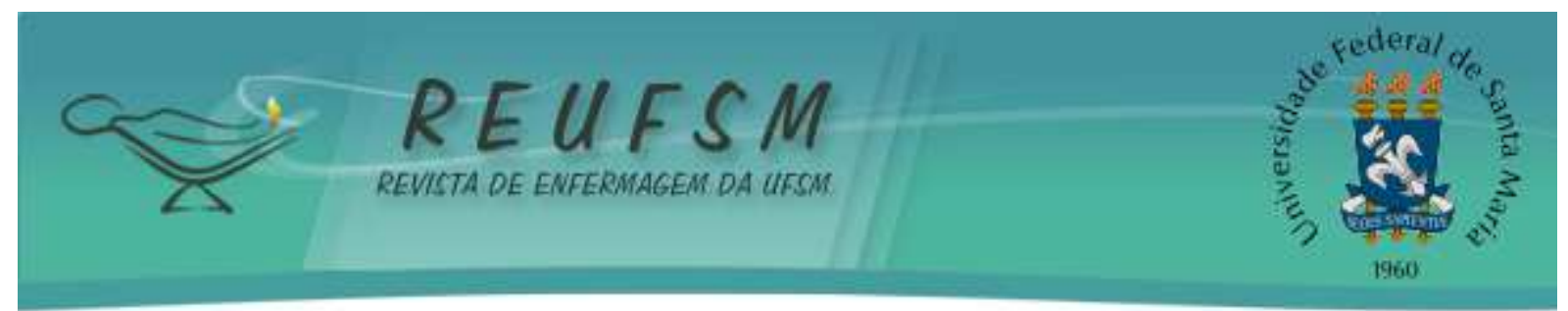

structure thereof, by poor or non-existent communication and lack of humanization in assistance. Conclusion: In the face of perceived difficulties in primary health care services, users seek care in hospitals, a situation that discourages the establishment and coordination of health care networks.

Descriptors: Nursing; Humanization of assistance; Comprehensive health care; Health services.

RESUMEN: Objetivo: Conocer la percepción de los pacientes hospitalizados sobre el atendimiento en los servicios de salud. Método: investigación cualitativa, descriptiva y exploratoria, realizada en una unidad de Clín ${ }^{7} i c a$ Quirúrgica, entre octubre y noviembre de 2014. Los datos fueron recolectados por medio de entrevistas semi-estructuradas con los usuarios hospitalizados y analizados a partir del análisis temático. Resultados: Los resultados fueron discutidos a partir de las categorías: el caminar en los servicios de salud; y las realidades de la atención primaria de salud y del hospital. Se identificó que los usuarios comprenden que la atención recibida en los servicios de salud se ve obstaculizada por la falta de estructura de esta, por una deficiente o inexistente comunicación y la falta de humanización en la asistencia. Conclusión: En medio a las dificultades identificadas en los servicios de atención primaria de salud, los usuarios buscan atención en los hospitales, situación que desfavorece el establecimiento y la articulación de las redes de atención de la salud.

Descriptores: Enfermería; Humanización de la atención; Atención integral de salud; Servicios de salud.

\section{INTRODUÇÃO}

A modificação nos estilos de vida e hábitos relacionados à saúde evidenciados nos últimos anos tem contribuído para o adoecimento da população, e os serviços, muitas vezes, são insuficientes em termos quantitativos e qualitativos para atender às necessidades de saúde da população. É nessa perspectiva que os usuários apontam aspectos relacionados ao atendimento que podem ser melhorados, como a humanização da assistência, prática considerada desafiadora nos serviços de saúde. ${ }^{1-2}$

Entre os cenários de atendimento à saúde, a Atenção Primária à Saúde (APS) é o local de atendimento preferencial ao usuário na Rede de Atenção à Saúde (RAS). Assim, tem como função ser o centro de comunicação com os demais pontos de atenção à saúde, com condições de ordenar os fluxos e contrafluxos de pessoas e de informações nas redes. ${ }^{3}$ Neste estudo será adotada a opção semântica APS, porque propõe uma estratégia de organização do Sistema Único de Saúde (SUS), um movimento de mudança do ciclo de Atenção Básica para o da APS, que aproxima a denominação brasileira do restante do mundo. ${ }^{3}$

A instituição hospitalar, outro cenário de atendimento à saúde, é um local que deveria trabalhar de forma integrada com a APS para atender às necessidades dos usuários que a procuram. Porém, o que se percebe, muitas vezes, é um sistema sem comunicação e desarticulado dos demais serviços de atenção à saúde. ${ }^{4}$ Diante disso, para a efetivação da RAS é fundamental uma comunicação eficaz e resolutiva nos diferentes pontos de atenção. A APS deve ser ordenadora do cuidado e responsável pela resolutividade da atenção aos usurários de seu território. Entende-se que uma comunicação eficaz entre os diferentes serviços contribui para a desfragmentação da rede, assim como uma efetiva participação dos trabalhadores no âmbito da atenção. 


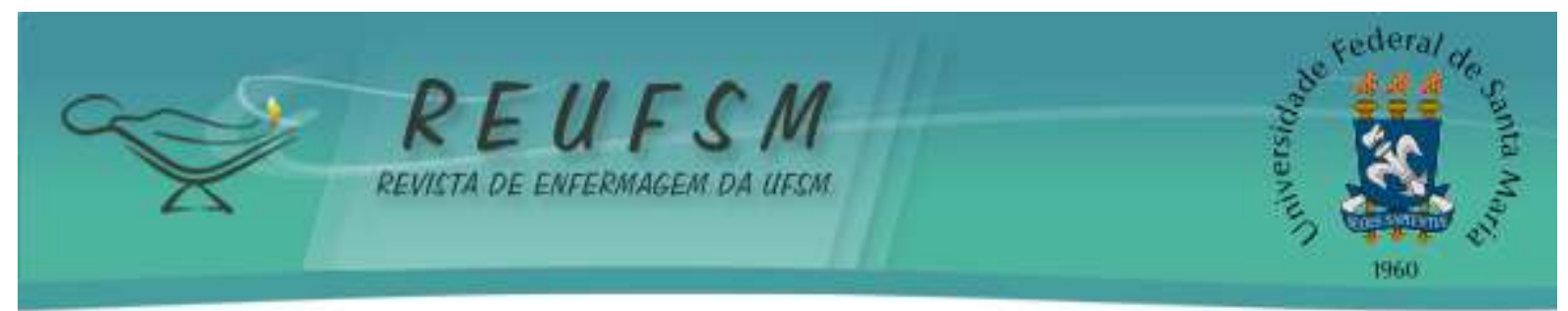

O enfermeiro é um profissional da equipe de saúde e possui como uma das suas atribuições previstas na Lei do Exercício Profissional realizar o planejamento do cuidado de forma individualizada e de acordo com as necessidades do usuário ${ }^{5}$. Tal planejamento implica na articulação e comunicação dos profissionais das diferentes densidades tecnológicas da RAS.

Observa-se, em muitas situações, dificuldades dos trabalhadores de saúde em formar essa rede de cuidados, especialmente entre a APS e o hospital. Essa dificuldade pode ser decorrente de diversos fatores, entre eles, fatores organizacionais e do próprio processo de trabalho em saúde.

Nessa perspectiva, avaliar os serviços de saúde na percepção dos usuários foi objeto de investigação em estudos. ${ }^{6,8}$ No entanto, não foram encontradas pesquisas que buscassem conhecer a compreensão de usuários hospitalizados sobre o atendimento recebido nos serviços de saúde anteriormente à internação, em especial na APS.

Essa temática se torna relevante na medida em que possibilita identificar aspectos do atendimento que precisam ser melhorados. A partir disso, novos estudos podem contribuir para as reflexões e produção de conhecimento na saúde e enfermagem e favorecer o controle social no âmbito do SUS, sendo importantes os processos de avaliação pelos usuários. ${ }^{7}$

Diante disso, emergiu o questionamento: como os usuários hospitalizados percebem o atendimento recebido nos serviços de saúde anteriores à internação? Este estudo objetivou conhecer a percepção de usuários hospitalizados sobre o atendimento nos serviços de saúde.

\section{MÉTODO}

Estudo qualitativo, do tipo descritivo-exploratório, realizado junto aos usuários internados em uma unidade de Clínica Cirúrgica de um hospital público, certificado como de ensino pelos Ministérios da Saúde e Educação. Esta instituição está localizada em um município do Estado do Rio Grande do Sul, sendo referência para 45 municípios da região centro-oeste do Estado, o que contempla uma população de mais de 1,15 milhão de habitantes, com atendimento de média e alta complexidade e pronto-socorro regional, atendendo exclusivamente usuários do SUS.

A referida unidade de internação presta assistência a usuários que se encontram nos períodos pré e pós-operatório de especialidades cirúrgicas (cirurgia geral/digestiva, urologia, traumatologia/ortopedia, cabeça e pescoço, vascular, proctologia). Optou-se por realizar o estudo neste cenário tendo em vista a realização da escuta qualificada junto aos usuários, na perspectiva da elaboração do projeto terapêutico singular. Ainda, a unidade de internação de clínica cirúrgica, local do estudo, abrange grande fluxo de usuários que são atendidos neste serviço. Sabe-se que esses usuários possuem uma experiência prévia de acesso a outros serviços de saúde e APS, anterior ao período de internação, o que instiga a investigar e aprofundar a percepção destes usuários no atendimento aos serviços de saúde.

Os critérios de inclusão dos participantes foram estar no período pré-operatório de qualquer especialidade cirúrgica e ter condições de expressão oral. Foram excluídos os usuários que não residiam no município sede da instituição.

A seleção dos participantes aconteceu de forma que cada especialidade cirúrgica fosse contemplada por, no mínimo, um usuário, até que o objetivo do estudo fosse alcançado. Assim, no período de outubro a novembro de 2014, no início do turno de trabalho da manhã (7 horas), os participantes eram identificados por meio do boletim de informações disponibilizado pela secretaria da unidade, em que constavam o nome, data 


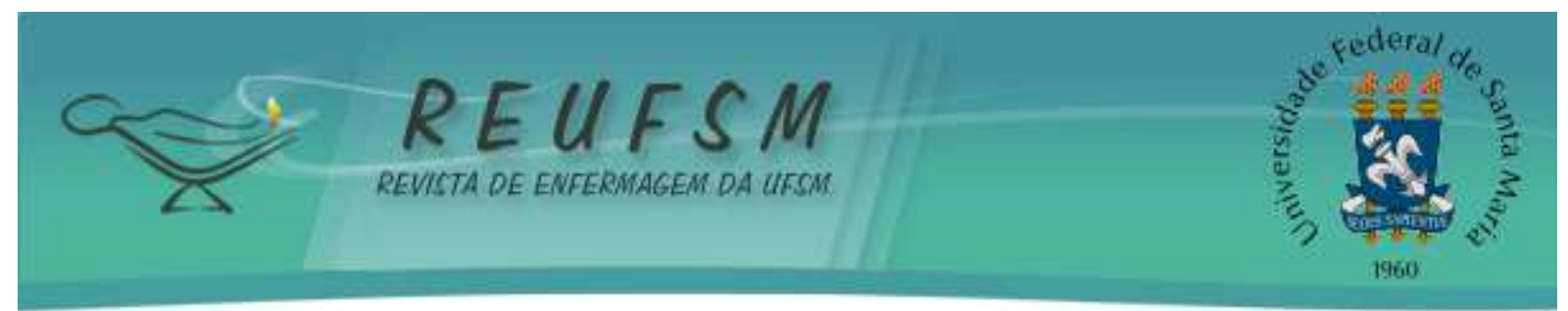

de nascimento, especialidade cirúrgica e período operatório. Posteriormente, realizava-se um sorteio manual dentre os elegíveis.

A técnica de coleta dos dados foi a entrevista semiestruturada, guiada por um roteiro com tópicos que serviram de orientação e guia para a interlocução. ${ }^{9}$ As entrevistas foram realizadas individualmente, em uma sala disponibilizada na unidade, na qual não haviam interferências externas e era oferecida privacidade ao participante. Tiveram duração média de 50 minutos, sendo audiogravadas mediante autorização do participante.

O critério utilizado para determinar a quantidade de entrevistas foi o de saturação amostral, que ocorre quando o conhecimento formado pelo pesquisador atingiu a homogeneidade, diversidade e intensidade das informações necessárias para atingir os objetivos do estudo. ${ }^{9}$ Assim, nove usuários participaram do estudo (cirurgia geral/digestiva - dois, urologia - dois, traumatologia/ortopedia - um, cabeça e pescoço - um, vascular dois, proctologia - um). Com o intuito de resguardar o anonimato dos participantes, as entrevistas foram identificadas pela palavra "Usuário", seguida de um número arábico sequencial, conforme a ordem de realização.

Os dados foram analisados por meio da Análise Temática, que consiste em descobrir os núcleos de sentido que compõem uma comunicação. Para auxiliar nessa interpretação, procurou-se seguir os passos: pré-análise, período em que ocorreram a leitura flutuante e a constituição do corpus das entrevistas, obtendo-se, assim, uma orientação para a análise do material empírico; exploração do material, período de categorização dos dados; tratamento dos resultados obtidos e interpretação. ${ }^{9}$

Este estudo obteve aprovação do Comitê de Ética em Pesquisa (Parecer 832.508 em 15 de outubro de 2014) e atendeu às prerrogativas da Resolução $n^{\circ} 466 / 12$ do Conselho Nacional de Saúde. Foi entregue e solicitada a leitura e assinatura do Termo de Consentimento Livre e Esclarecido, ficando uma via de posse do participante. Também foi utilizado o Termo de Concordância para a gravação de voz.

Destaca-se que, entre os usuários convidados para participar do estudo, dois se recusaram e um usuário desistiu após conversar particularmente com familiar e não achar interessante a temática da pesquisa. Assim, foi realizado novo sorteio para compor a amostra. Nesta pesquisa, assegurou-se o princípio da autonomia do participante, o qual consiste em considerar a participação de modo voluntário, sem coerção institucional ou psicológica.

\section{RESULTADOS}

Participaram do estudo nove usuários, seis homens e três mulheres, com idade entre 35 e 69 anos. A partir da análise dos dados, emergiram duas categorias temáticas: o caminhar nos serviços de saúde; e realidades da Atenção Primária à Saúde e da hospitalar.

\section{O caminhar nos serviços de saúde}

Nesta categoria, os participantes mencionaram a falta de informação, a comunicação deficiente e a burocracia nos serviços de saúde como os aspectos que influenciaram o caminhar deles nos serviços de saúde:

[...] olha, eu estava perdido, fui na UPA (Unidade de Pronto Atendimento), me mandaram para casa, voltei para a UPA com dor, fizeram $R X$ e me encaminharam aqui para o hospital. Ninguém me disse que era para fazer cirurgia [...], tratado como bicho. (Usuário 5) 


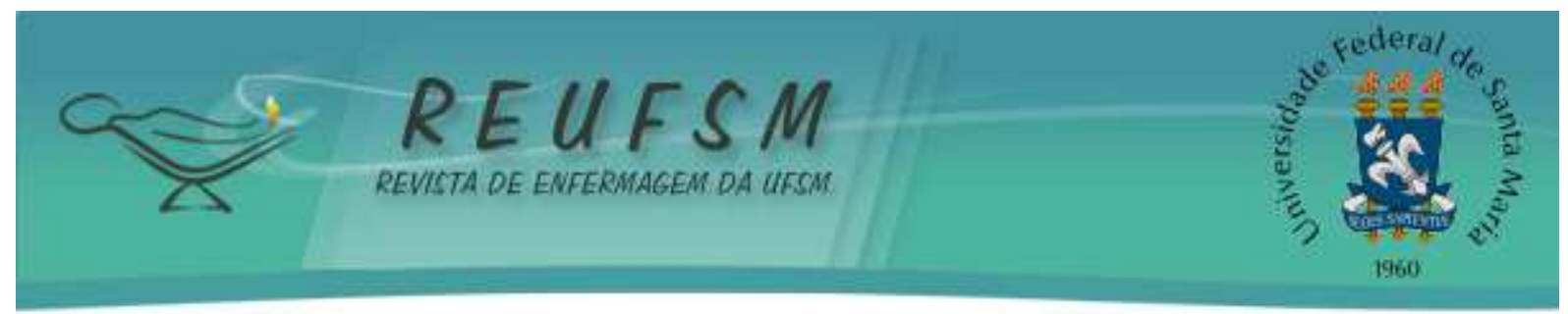

[...] sinceramente eu não entendo porque tanta papelada, se eu já faço tratamento aqui (referindo-se à instituição hospitalar) por que eu tenho que voltar no posto e pegar um novo encaminhamento? (Usuário 7)

O encaminhamento do usuário nos serviços da rede do município depende, essencialmente, da articulação dos profissionais da saúde. Assim, a resolutividade da atenção à saúde depende de um sistema em que a comunicação seja eficaz e desfragmentada, caso contrário compromete a eficácia e qualidade do atendimento prestado à população. Os relatos, a seguir, retratam esses aspectos:

[...] o médico do posto só me encaminhou, não falou nada do que poderia acontecer comigo aqui no hospital [...] eles explicam mal sobre minha cirurgia [...] vão tirar um pedaço do estômago ou tirar todo [...] eles não têm certeza? (Usuário 1)

[...] veio uma doutora (referindo-se ao atendimento no posto de saúde) que não me examinou em nada, só perguntou o que eu estava sentindo e mandou fazer em mim uma injeção e disse que era da coluna a dor [...]. (Usuário 2)

Orientar e escutar o usuário são deveres dos profissionais da saúde, um dos requisitos essenciais na direção da humanização do atendimento na saúde:

[...] o médico do posto me explicou que eu não precisava pagar nenhum exame, tudo pelo SUS [...], e graças a ele eu descobri cedo o tumor que apareceu na tomografia. (Usuário 6)

Há usuários inconformados com a superlotação enfrentada nos serviços de saúde. Assim, percorrem caminhos que comprometem, por vezes, o fluxo da RAS:

[...] não sei o que estavam esperando, se era falta de leito, má vontade [...], mas só foi meu irmão entrar na justiça, que no outro dia já tinha leito vago aqui no hospital. (Usuário 8)

[...] hoje eu estou aqui no hospital porque minha sobrinha trabalha como instrumentadora e conhece a médica [...], foi ela quem conseguiu agilizar para mim. (Usuário 9)

\section{Realidades da Atenção Primária à Saúde e da hospitalar}

A impessoalidade nos atendimentos da APS é percebida pelos usuários como uma dificuldade:

[...] o médico (referindo-se ao atendimento no posto de saúde) não olha para a gente, atende de cabeça baixa. Isso é uma dificuldade [...], eu com duas hérnias de disco, com dor, eles receitam só paracetamol [...]. (Usuário 1) 


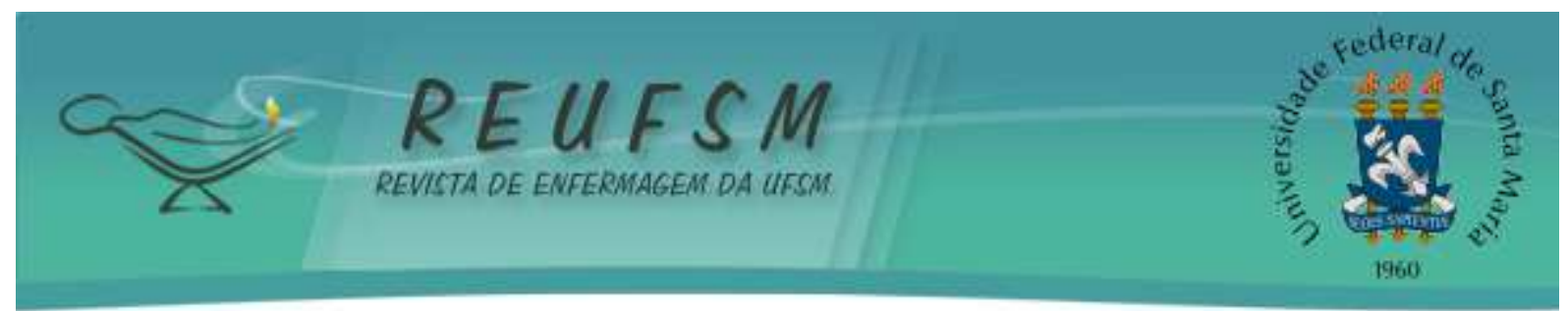

[...] olha, tem uns doutores naquele posto que nem deixam tu te deitares na mesa, já vão pegando papel e vão escrevendo como se adivinhassem o que a gente tem. (Usuário 4)

Os usuários percebiam a atuação multiprofissional efetivada na instituição hospitalar como uma estratégia positiva na continuidade dos cuidados nos serviços de saúde:

[...] na internação domiciliar só fui melhorando, [...] ia médico, enfermeiro, estagiário, nutricionista, fisioterapeuta, [...] isso foi muito bom [...] estou bem orientado, a equipe médica e depois a de enfermagem me explicaram tudo direitinho sobre a cirurgia. Aqui no hospital o pessoal é muito bom mesmo, [...] já virou uma amizade. (Usuário 3)

[...] muito bom o atendimento aqui no hospital, dos médicos, enfermeiros, muito bom mesmo! Tu percebes que aqui te escutam de verdade. No posto não dá nem para falar. (Usuário 4)

A falta de resolutividade na APS, percebida pelos participantes deste estudo, caracteriza a realidade enfrentada diariamente pelos usuários do SUS, o que pode acarretar em sobrecarga de alguns serviços e aumentar a procura pelo serviço hospitalar:

[...] eu não vou ao posto perto de casa, vou na UPA direto [...], lá fazem o exame que tiver que fazer [...], demora para ser atendido, é verdade, fazer o que [...], o posto perto de casa é uma vergonha, falta tudo! (Usuário 5)

[...] eu não sei se só posso consultar no postinho perto de casa [...] a gente vai onde atendem a gente [...] e lá está demorando um horror para o retorno com o especialista. (Usuário 4)

O usuário percebe as dificuldades dos serviços de saúde e, por vezes, compara os atendimentos na APS e no hospital:

[...] os vândalos destruíram o posto, não tem guarda, [...] não tem agente comunitário para acompanhar a gente, [...] as enfermeiras se viram lá dentro para dar conta. (Usuário 1)

[...] a gente tem que ir direto na Secretaria de Saúde se quiser que o exame saia rápido, lá mesmo no posto eles falam pra gente ir na Secretaria para dar uma pressionada no pessoal [...]. (Usuário 4)

[...] eu acho que vou continuar meu tratamento aqui (referindo-se ao hospital), gostaria que fosse, tem mais recursos [...], lá no posto não tem nada, não tem condições, tem muita gente, acho que para o curativo tem [...] e não me falaram nada se eu ia voltar para me tratar lá. (Usuário1) 


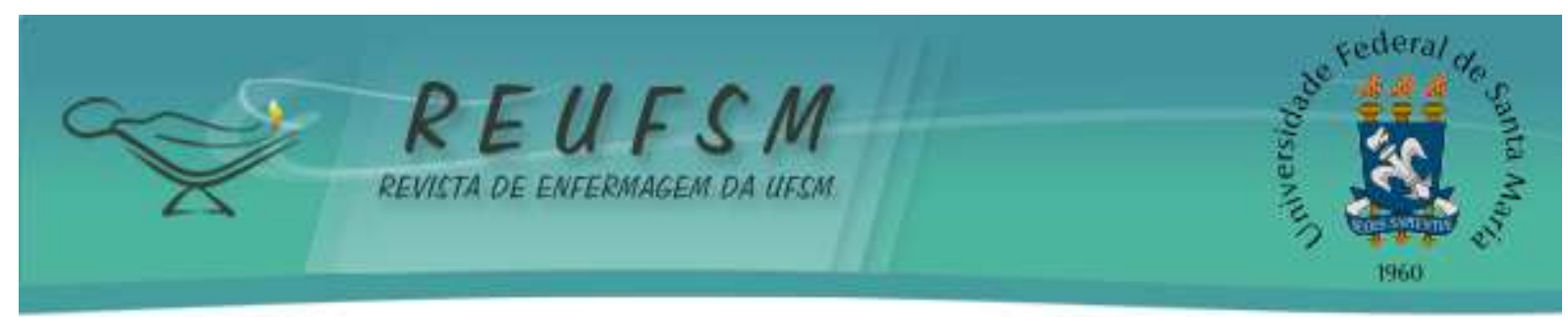

[...] gostaria de continuar aqui meu tratamento (referindo-se ao hospital), porque se fosse para ir para o posto já tinha morrido faz muito tempo. (Usuário 2)

Os relatos dos participantes demonstram a percepção de que faltam recursos na APS, o que retarda ou inviabiliza o atendimento de suas necessidades de saúde. Além disso, a organização das RAS faz com que o usuário necessite percorrer um caminho mais longo na APS ou recorra a serviços hospitalares em busca de atendimento.

\section{DISCUSSÃO}

Por meio dos relatos, percebe-se o desejo de que o atendimento às necessidades de saúde fosse realizado em especial pela instituição hospitalar, pois os participantes do estudo consideram um local que possui recursos para os procedimentos e que oferece escuta atenta. É importante destacar que a finalidade de conhecer a percepção de usuários hospitalizados sobre o atendimento nos serviços de saúde consiste na compreensão sobre os fatores reais que fazem com que os usuários superlotem os serviços hospitalares em busca de atendimento. Sabe-se da existência da referência e contrarreferência entre os serviços, fato não mencionado pelos participantes deste estudo.

Assim, o repertório de fatores envolvidos no acesso aos serviços de saúde aponta para a complexidade da conformação e concretização da RAS. Essas são organizações poliárquicas de conjuntos de serviços de saúde vinculadas entre si por uma missão única, por objetivos comuns e por uma ação cooperativa e interdependente, que permite ofertar uma atenção contínua e integral à determinada população. ${ }^{3}$

Na rede, um dos papéis da APS é orientar o cuidado ao longo de todos os pontos de atenção e de toda a vida de uma comunidade.$^{10} \mathrm{~A}$ relação médico-paciente, no contexto da atenção primária, constitui-se em um dos desafios para o estabelecimento de práticas humanizadas em saúde. ${ }^{11}$

A APS é a via preferencial de entrada do sistema, com vistas a organizar o processo de trabalho de equipes multiprofissionais na perspectiva de abordagem integral do processo saúde/doença. Da mesma forma, visa garantir o acesso de cada usuário a qualquer outra unidade do sistema em função das suas necessidades. ${ }^{3}$

Neste estudo, percebe-se que a falta de informação para os usuários se constitui em um dos elementos primordiais que precisa de investimento para garantir a humanização nos serviços. Sobre isso, cita-se a Política Nacional de Humanização ${ }^{12}$, a qual contempla um conjunto de ações que têm como objetivo a construção de um novo posicionamento dos serviços prestados, centrado na recuperação das dimensões de um cuidado humanizado de assistência à saúde dos usuários, que é mais amplo do que o tratamento centrado na doença. A proposta de humanização pode ser entendida como um conjunto de princípios e diretrizes, que reafirmam a valorização dos diferentes sujeitos implicados no processo de produção de saúde (usuários, trabalhadores e gestores).

Nesse contexto, o atendimento que prioriza a escuta do usuário propicia o vínculo do binômio usuário-serviço de saúde, o que pode humanizar a assistência. Neste estudo, identificaram-se a falta de informações por parte de alguns profissionais da saúde com relação ao usuário e uma comunicação ineficaz entre ambos os sujeitos.

Esse resultado diverge do encontrado em estudo realizado com usuários do SUS em um município do Rio de Janeiro, o qual identificou como satisfatórios elementos constituintes das relações entre os sujeitos, sendo esse um dos melhores itens avaliados. Ainda, na pesquisa, avaliou-se como efetiva a dimensão da informação e comunicação, com destaque positivo no que se refere à explicação do profissional sobre exames e 


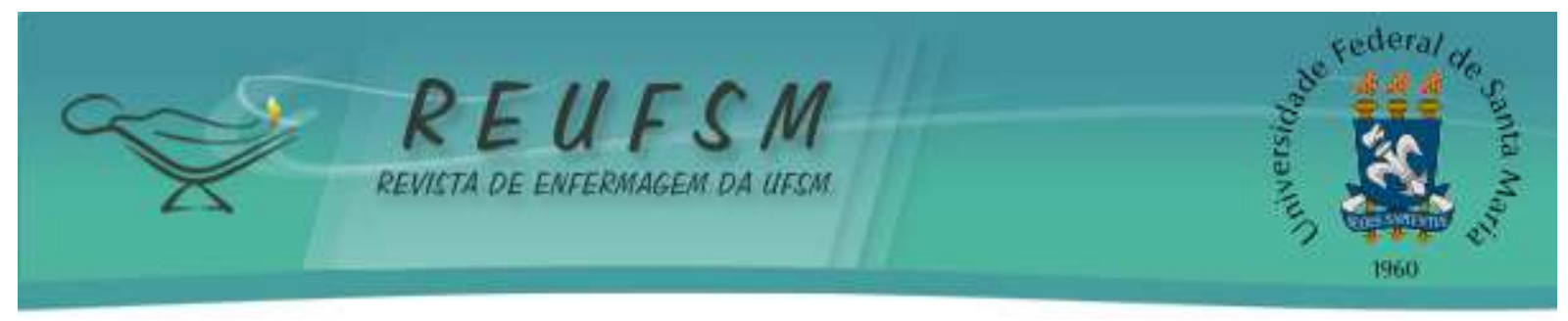

sintomas da doença. Tais elementos corroboram para a humanização da assistência aos usuários, destacando o esclarecimento do profissional, acolhimento e escuta qualificada. ${ }^{8}$

As relações entre os profissionais de saúde e os usuários são um desafio para a reorganização do SUS. A comunicação é um dos elementos essenciais para a superação desse desafio, pois auxilia na organização dos serviços de saúde e contribui para a continuidade e adesão ao tratamento sendo necessário, para isso, reconhecer os usuários como pessoas com direito à cidadania, dignas de respeito e singularização. ${ }^{1}$

Promover a saúde é, também, instituir relações acolhedoras de cuidado entre trabalhadores de saúde e usuários dos serviços de saúde. Essas relações implicam em garantia dos direitos de cidadania, redução dos riscos à saúde e da vulnerabilidade às doenças e agravos, o que remete à necessidade de organização e gestão do trabalho que reconheçam e respeitem a singularidade dos atores sociais da produção de saúde, reduzindo-se às desigualdades. ${ }^{13}$

Os usuários em condição de vulnerabilidade são os que precisam ser cuidados, especialmente na APS, com vistas a diminuir as desigualdades sociais. Essa perspectiva necessita ser contemplada nos processos educativos em saúde e incorporada como tema de reflexão permanente por parte dos profissionais. ${ }^{11}$

A humanização na saúde propõe uma construção coletiva que só pode acontecer a partir da troca de saberes, por meio do trabalho em rede com equipes multiprofissionais, da identificação das necessidades, desejos e interesses dos envolvidos. Além disso, há necessidade do reconhecimento de gestores, trabalhadores e usuários como sujeitos ativos e protagonistas das ações de saúde, de condições de trabalho e da criação de redes solidárias e interativas, participativas e protagonistas do SUS.

Assim, é necessário oferecer aos trabalhadores de saúde condições mínimas, com o objetivo de efetivar as ações de saúde, o que possibilita o alcance dos objetivos traduzidos pela pactuação da atenção básica, com a finalidade de atender às necessidades de cada localidade. ${ }^{14} \mathrm{O}$ enfermeiro estabelece um papel de gestor e articulador das RAS, podendo ordenar e compartilhar conhecimentos com outros trabalhadores no intuito de fortalecer as redes.

$\mathrm{Na}$ fala dos participantes deste estudo, observa-se a preferência pelo sistema hospitalar de atenção à saúde. Nessa direção, pesquisa sobre o perfil de usuários frequentes em um serviço de emergência evidenciou que apesar de os usuários utilizarem a APS e a atenção especializada de forma contínua, recorrer ao serviço hospitalar se constitui em uma estratégia para a obtenção de assistência rápida. Além disso, a busca de atendimento de emergência se relacionou a aspectos como organização do sistema de saúde, facilidade de acesso, percepção de maior resolutividade, disponibilidade de tecnologia, formação de vínculo e retornos agendados. ${ }^{15}$

Ainda, no que se refere à preferência pelo sistema hospitalar de atenção à saúde, a qualidade da assistência na internação domiciliar foi outro aspecto mencionado pelos participantes desta pesquisa. Autores consideram que o domicílio é um ambiente dinâmico, no qual a complexidade perpassa todas as modalidades de assistência que constituem demandas a serem trabalhadas na formação profissional. 0 enfermeiro, nesse cenário, possui papel fundamental de atuação, pois, além de prestar cuidados diretos, gerenciar o processo de trabalho da equipe de enfermagem e participar do treinamento do cuidador familiar, tem como funções a elaboração e gestão do plano de cuidados dos usuários, responsabilizando-se por mobilizar outros trabalhadores para a assistência ${ }^{16}$, sugerindo uma atuação multiprofissional e interdisciplinar.

Além disso, percebe-se a necessidade de equipes multiprofissionais na APS. 0 trabalho em equipe pode superar a fragmentação do sistema e contribuir para o 


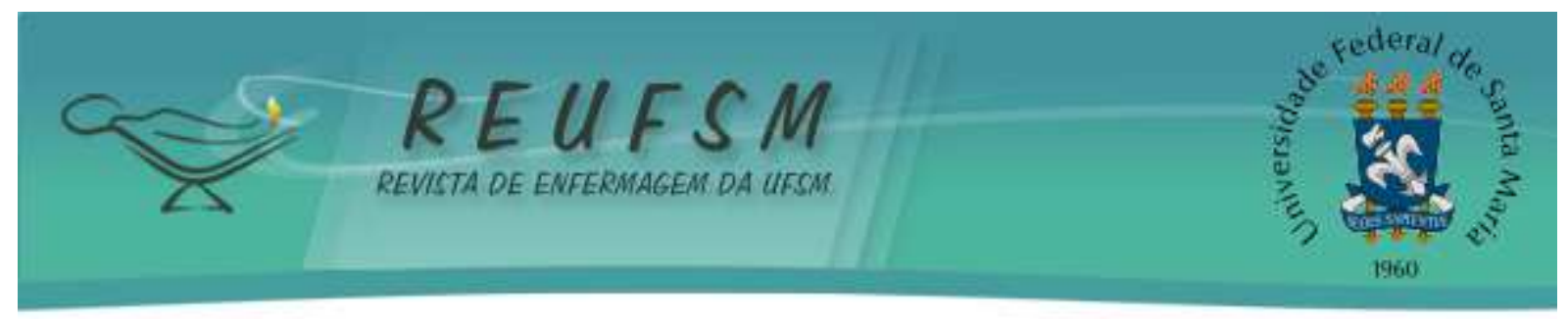

estabelecimento da RAS com reordenação das ações de atenção, no intuito de abranger as diferentes necessidades dos usuários.

O serviço de internação domiciliar tem, juntamente com a equipe multiprofissional, o objetivo principal de encaminhar o usuário para a APS mais próxima de sua residência, objetivando reestabelecer a sua saúde. Porém, são encontradas falhas na comunicação entre a APS e a instituição hospitalar, evidenciando deficiências nos serviços de saúde ${ }^{17}$. A partir disso, reforça-se a importância do enfermeiro como profissional que pode desencadear ações de planejamento e gerenciamento do cuidado, tanto em rede de APS como em âmbito hospitalar, contribuindo, assim, para a desfragmentação da rede.

Refletir sobre as condições e as relações de trabalho presentes na APS e no hospital é pensar sobre um dos aspectos centrais da Política de Humanização, que destaca que a baixa qualidade dos serviços sobrecarrega o sistema, isso porque se o trabalhador de saúde tem dificuldade na realização de seu trabalho, o usuário fica insatisfeito com 0 atendimento. Assim, o gestor não consegue ter uma boa comunicação e interação com outras instâncias, os encaminhamentos ficam desorganizados e geram um número considerável de consultas, exames complementares e internações adicionais. ${ }^{12}$

Nessa direção, reafirma-se que a grande demanda do serviço é um dos aspectos que prejudica a humanização do atendimento ${ }^{4}$. A proposta da humanização nos serviços de saúde também é desfavorecida por aspectos relacionados à falta de comunicação, carga horária de trabalho excessiva e problemas institucionais. ${ }^{18}$ Para humanizar a assistência é preciso acolher, escutar e respeitar o outro.

A humanização nas instituições de saúde surge para que o trabalhador possa refletir sobre o seu cotidiano laboral, dando um novo sentido para o atendimento. ${ }^{2}$ Assim, almejase, além da resolutividade dos serviços, uma interação entre usuário e trabalhadores, que favoreça o estabelecimento de um vínculo.

Essa proposta de interação e diálogo é essencial para a construção da humanização, pois a capacidade de falar e ouvir perpassa as questões de empatia e valorização dos diferentes sujeitos. ${ }^{19}$ Pensar nas relações de empatia é se posicionar no lugar do outro, transformando as questões relativas ao cuidar, correlacionado, assim, a fatores da subjetividade dos mesmos. ${ }^{18}$

Nos serviços de saúde é preciso ter condições de trabalho que permitam ser e estar sensível às demandas, a partir de uma escuta qualificada, do conhecimento e entendimento da realidade do usuário. Isso pode favorecer um planejamento adequado da assistência, bem como a implementação de ações que contemplem as necessidades de maneira integral.

Assim, reafirma-se a necessidade de instrumentalizar o usuário sobre seus direitos, oferecer condições de trabalho satisfatórias e ampliar o diálogo no que se refere ao serviço oferecido pela APS e hospitalar e entre os trabalhadores que prestam o atendimento. Esses aspectos se constituem em passos importantes para qualificar e solidificar a RAS.

\section{CONSIDERAÇÕES FINAIS}

Os usuários hospitalizados percebem que o atendimento recebido nos serviços de saúde, anteriormente à internação, é dificultado. As dificuldades se relacionam à falta de estrutura dos serviços, à comunicação deficiente ou inexistente e à falta de humanização na assistência.

$\mathrm{Na}$ percepção dos usuários, a procura pelo atendimento na instituição hospitalar pode ser mais resolutiva quando comparada com a APS, situação que pode contribuir para aumentar as filas de espera por atendimento nos hospitais. Com isso, ratifica-se a necessidade de fornecer ao usuário informações no que se refere aos serviços oferecidos 


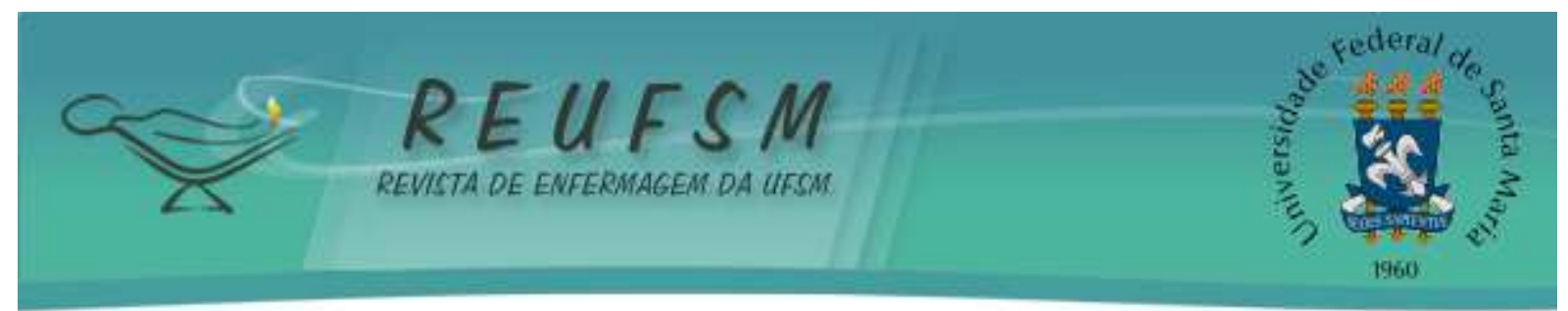

pela RAS, o que poderá contribuir para minimizar as filas no atendimento em serviços com maior densidade tecnológica e fortalecer a rede.

Menciona-se, como possível limitação, o fato de o estudo retratar uma realidade locorregional. Portanto, tal limitação remete à necessidade de se investigar a temática em outros cenários.

Assim, como são os usuários quem usufruem dos serviços, acredita-se que o conhecimento produzido poderá suscitar reflexões de trabalhadores e gestores que se insiram nos espaços das RAS, e subsidiar o planejamento e desenvolvimento de ações que reorientem as suas práticas, com vistas a qualificar os serviços, além de contribuir para a construção do conhecimento sobre a temática. Nesse sentido, os resultados reafirmam a importância do enfermeiro no processo de orientação e condução do usuário nos diferentes pontos de atenção à saúde.

A pesquisa também contribui para a reflexão dos enfermeiros docentes e assistenciais, que atuam em instituições de ensino na condução do processo de aprendizado dos futuros profissionais da saúde.

Esse processo necessita estar fundamentado no conhecimento das políticas públicas e nas formas de articulação da rede, o que requer engajamento profissional que culmine em responsabilização pelos atos de ensinar e cuidar.

\section{REFERÊNCIAS}

1. Schimith MD, Simon BS, Brêtas ACP, Budó MLD. Relações entre profissionais de saúde e usuários durante as práticas em saúde. Trab Educ Saúde. 2012;9(3):479-503.

2. Cavalcante AKCB, Damasceno CAF, Miranda MDS. Humanização da assistência em atendimento de urgência hospitalar: percepção dos enfermeiros. Rev Baiana Enferm. 2013;27(3):221-33.

3. Mendes EV. As redes de atenção à saúde. 2. ed. Brasília: Organização Pan-Americana da Saúde; 2011.

4. Duarte MLC, Noro A. Humanização do atendimento no setor de radiologia: dificuldades e sugestões dos profissionais de enfermagem. Cogitare Enferm. 2013;18(3):532-8.

5. Brasil. Lei n. 7.498, de 25 de junho de 1986. Dispõe sobre a Regulamentação do Exercício da Enfermagem e dá outras providências. Diário Oficial da República Federativa do Brasil, Brasília; 1986 jun 26. Seção 1:1.

6. Baraldi DC, Souto BGA. A demanda do acolhimento em uma Unidade de Saúde da Família em São Carlos, São Paulo. Arq Bras Ciênc Saúde. 2011;36(1):10-7.

7. Arakawa AM, Herrera SAL, Caldana ML, Tomita, NE. Percepção dos usuários do SUS: expectativas e satisfação do atendimento na estratégia de saúde da família. Rev CEFAC. 2012;14(6):1108-14.

8. Brandão ALRBS, Giovanella L, Campos CEA. Avaliação da atenção básica pela perspectiva dos usuários: adaptação do instrumento EUROPEP para grandes centros urbanos brasileiros. Ciênc Saúde Coletiva. 2013;18(1):103-14.

9. Minayo MCS. O desafio do conhecimento: Pesquisa qualitativa em saúde. $14^{\mathrm{a}}$ ed. São Paulo: HUCITEC; 2014.

10. Organização Pan-Americana Da Saúde (OPAS). A atenção à saúde coordenada pela APS: construindo as redes de atenção no SUS: contribuições para o debate. Brasília: OPAS; 2011. (NAVEGADORSUS; 2 ). 


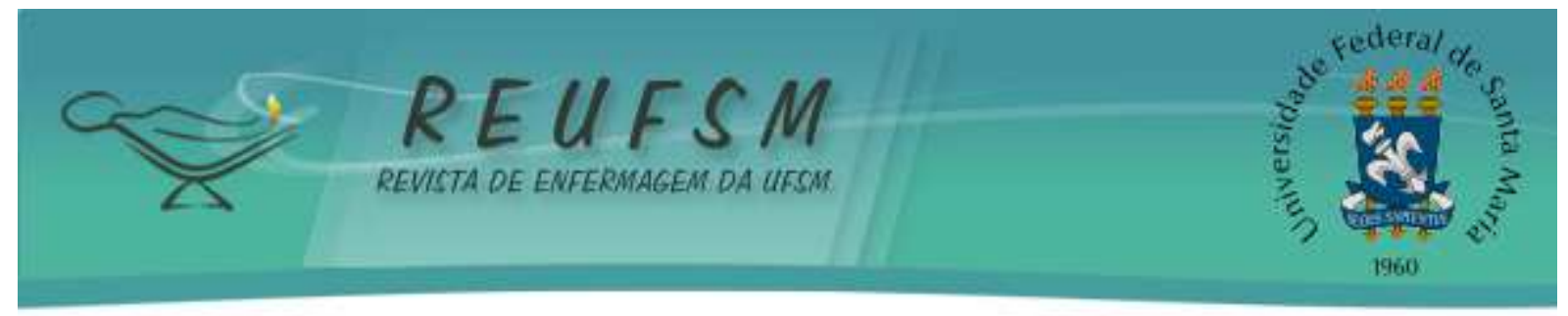

11. Gomes AMA, Caprara A, Landim LOP, Vasconcelos MGF. Relação médico-paciente: entre o desejável e o possível na Atenção Primária à Saúde. Physis. 2012;22(3):1101-19.

12. Brasil. Ministério da Saúde. HumanizaSUS. Política Nacional de Humanização: a humanização como eixo norteador das práticas de atenção e gestão em todas as instâncias do SUS. Brasília (DF): Ministério da Saúde; 2004. (Série B. Textos Básicos de Saúde).

13. Brasil. Ministério da Saúde. Política Nacional de Promoção da Saúde. Brasília (DF): Ministério da Saúde; 2006. (Série B. Textos Básicos de Saúde).

14. Silva LMS, Mendes EP, Torres RAM. Trabalho interdisciplinar na estratégia saúde da família: enfoque nas ações de cuidado e gerência. Rev Enferm UERJ. 2012;20(Esp 2):784-8.

15. Acosta AM, Lima MADS. Frequent users of emergency services: associated factors and reasons for seeking care. Rev Latinoam Enferm [Internet]. 2015 abr [acesso em 2016 ago 24];23(2):337-44. Disponivel em: http://www.scielo.br/pdf/rlae/v23n2/0104-1169-rlae-2302-00337.pdf.

16. Silva LK, Sena RR, Silva PM, Souza CG, Martins ACS. Atuação do enfermeiro nos serviços de atenção domiciliar: implicações para o processo de formação. Ciênc Cuid Ssaúde. 2014;13(3):503-10.

17. Engel RH, Weiller TH, Brondani CM. Inserção da residência multiprofissional em um serviço de internação domiciliar: atuações de enfermagem. Rev Contexto \& Saúde. 2011;10(20):1037-40.

18. Chernicharo IM, Silva FD, Ferreira MA. Caracterização do termo humanização na assistência por profissionais de enfermagem. Esc Anna Nery Rev Enferm. 2014;18(1):15662.

19. Lima Neto AVL, Nunes VMA, Fernandes RL, Barbosa IML, Carvalho GRP. Acolhimento e humanização da assistência em pronto-socorro adulto: percepções de enfermeiros. Rev Enferm UFSM [Internet]. 2013 [acesso em 2016 abr 10];3(2):276-86. Disponível em: http>// http://periodicos.ufsm.br/reufsm/article/view/8279/pdf.

Data de recebimento: $23 / 05 / 2016$

Data de aceite: 20/12/2016

Contato com autor responsável: Pâmela Guimarães Siqueira

Endereço postal: Rua Belo Horizonte, número 205, Bairro Parque Pinheiro Machado, CEP: 97.030-060 - Santa Maria, rs- Brasil. E-mail: pamelaguimaraessiqueira@gmail.com 